\title{
Realistic Simulation of Synthetic Images on Computer Monitors Based on the Color Appearance Model CIECAM02
}

\author{
Vladimir Budak $^{1,2}$ and Ekaterina Emelianova ${ }^{2}$ \\ ${ }^{1}$ National Research University "MPEI", Krasnokazarmennaya 14, Moscow, 111250, Russia \\ ${ }^{2}$ National University of Science and Technology "MISiS”, Leninskiy Prospekt 4, Moscow, 119991, Russia
}

\begin{abstract}
The theory of global illumination and computer programs based on it allows calculating the light field accurately in an arbitrary three-dimensional lighting scene. However, the output of the visualization of the light field spatial-angular distribution to the display screen in the form of an image is inevitably associated with scaling the luminance and color of the image pixels to the computer display dynamic range. To date, a color appearance model was created in colorimetry. This model allows recalculating the image pixels' color for viewing conditions other than the original ones preserving the image's visual perception. This model is approved by the CIE (International Commission on Illumination) as a standard model under the name CIECAM02. In this paper, the CIECAM02 algorithm model is implemented, and a study of designing a lighting system for lighting a theater stage to create an atmosphere of sunset is carried out. Modeling the lighting system is performed in the DIALux evo program, which is the de facto standard of design in lighting engineering. The correspondence of the visualization of the stage lighting to the feeling of sunset is analyzed based on expert assessments. The research allows us to recommend the inclusion of the CIECAM02 model in the algorithms for visualizing the image of three-dimensional lighting scenes.
\end{abstract}

\section{Keywords}

Realistic modeling, Chroma adaptation transform, Color appearance model, CIECAM02.

\section{Introduction}

The theory of global illumination allows us to strictly calculate the light field in the lighting scene, considering all the effects in the ray photometric approximation. The calculation of the light field allows generating a realistic synthetic image of the scene from the camera at any point of the scene. However, when displaying this image on the monitor screen, a transition from the real luminance of the light field of a three-dimensional scene to the dynamic range of screen luminance is inevitable. It changes the luminance and color adaptation of the computer user's eye, which inevitably makes the perception of the resulting synthetic image different from the perception of the actual scene. From strength, speech can go only about a photorealistic image.

Currently, the mathematical model CAT/CAM (Chroma adaptation transform/ Color appearance model) has been developed in colorimetry, which allows describing the perception of an image by the eye based on stimuli (measurement and description of color) and the synthesis of stimuli based on perceptions (color reproduction). Thus, the laws of light-color similarity of images are determined, which preserve its perception when the conditions of luminance and color adaptation change. In 2002, the CIE (International Commission on Illumination, by the abbreviation of the French name Commission Internationale de l'éclairage) adopted the CIECAM02 standard [1,2]. Creating virtual reality on the computer was the leading topic during the 29th Quadrennial Session of the CIE in Washington in 2019, for example [3, 4]. The CIECAM16 standard model is currently being discussed but has not yet been adopted [5].

GraphiCon 2021: 31st International Conference on Computer Graphics and Vision, September 27-30, 2021, Nizhny Novgorod, Russia EMAIL: budakvp@gmail.com (V. Budak); emelyanovakatevlad@gmail.com (E. Emelianova) ORCID: 0000-0003-4750-0160 (V. Budak); 0000-0003-4537-3567 (E. Emelianova)

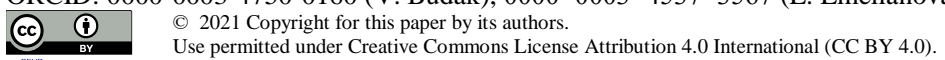

Use permitted under Creative Commons License Attribution 4.0
CEUR Workshop Proceedings (CEUR-WS.org) 
This article aims to implement the CIECAM02 model and test it for stage lighting to create a realistic sunset atmosphere on the stage. Based on expert assessments, the requirements for lighting that creates an atmosphere of sunset are determined. Then, in the standard program for designing lighting installations (LI), a corresponding model is created, based on modeling results in which a synthetic image of this LI is generated. Next, the resulting image is converted according to the CIECAM02 model to a realistic one. By the method of the expert assessments, one determines the discrepancy between the image and the sunset, and appropriate changes are introduced into the LI model. Consequently, we get a model of the LI, which implements the sunset impression on the stage in the lighting.

\section{Color appearance model CIECAM02}

Thanks to the works of Newton [6], Young [7], Helmholtz [8], Grassmann [9], and Maxwell [10], three-color colorimetry was created, which proved the vector method of adding unrelated colors. In representing unrelated colors, the perception of any isolated stimulus can be compared with three main colors. Tricolor colorimetry was standardized by the CIE in 1931 [11]. The system has become very popular because it provides a basis for measuring and reproducing color. However, three-color colorimetry is not completely satisfactory since the vector description of color does not consider chromatic induction, chromatic adaptation, and color constancy.

The CIECAM02 color appearance model allows determining color perception when observing conditions change. The color sensation in CIECAM02 is defined in terms of lightness, saturation, and color tone [2]:

- Brightness $(J)$ is an attribute of a visual sensation according to which an area appears to emit more or less light.

- Chroma $(C)$ is an attribute of a visual sensation according to which the perceived color of an area appears to be more or less chromatic.

- Hue $(H)$ is an attribute of a visual sensation according to which an area appears to be similar to one of the perceived colors: red, yellow, green, and blue, or to a combination of two of them.

Images that have the full set of JCH equal are perceived as identical.

Input data for the CIECAM02 include [1]:

- the relative tristimulus values of the test stimulus $(X Y Z)$;

- the relative tristimulus values of the white point $\left(X_{w} Y_{w} Z_{w}\right)$;

- the adapting luminance (often taken to be $20 \%$ of the luminance a white object in the scene)

$L_{A}$, in $c d / m^{2}$;

- the relative luminance of the surround (dark, dim, average);

- a decision on whether discounting-the-illuminant is taking place.

The surround relative luminance is generally taken to be average for reflection prints, dim for CRT displays or televisions, and dark for projected transparencies under the assumption that these media are being viewed in their typical environments. Discounting-the-illuminant is generally assumed to occur for object color stimuli such as prints and not to occur for emissive displays.

Chromatic adaptation is the human visual system's ability to adjust to illumination changes to preserve the appearance of object colors. It is responsible for the regular appearance of object colors despite the wide variation of light reflected from an object and observed by our eyes. The chromatic adaptation transform (CAT) system emulates this critical aspect of color perception in color appearance models (CAM).

All viable modern chromatic adaptation models can trace their roots, both conceptually and mathematically, to the hypotheses of Johannes von Kries [12]: "This can be conceived in the sense that the individual components present in the organ of vision are completely independent of one another and each is fatigued or adapted exclusively according to its own function."

The color-sensitive receptors of the human retina are cones of three types are the most properly referred to as $L, M$, and $S$ cones. These names refer to the long-wavelength, middle-wavelength, and short-wavelength sensitive cones, respectively. Sometimes the cones are denoted with other symbols such as $R G B$ suggestive of red, green, and blue sensitivities. As seen from their spectral responsivities, this concept is erroneous, and the $L M S$ names are more appropriately descriptive. 
Under the $R G B$ color model, we will, as usual, denote an additive color model in which the three main colors (red, green, and blue) of light are combined to reproduce the real color. The $R G B$ model is additive, where colors are obtained by adding to the black color. The $R G B$ color model is device dependent. All pixels in the image file are recorded in $R G B$ format for a standard computer graphics monitor $s R G B$. Accordingly, in working with images on a computer, the source data will be the colors in the $R G B$ system.

All colors in the CIECAM02 system change in the range [0,100]. However, since we will implement this model in the framework of MATLAB, where the color in floating-point format changes in the range $[0,1]$, then all expressions are written further for this range. At the same time, for the white point in the image file $R_{w}=G_{w}=B_{w}=1.0$.

Since according to the von Kries hypothesis, the linear adaptation model applies to $L M S$-cones. The first step of the model is the transition from the $R G B$ values in the image to the values in the $L M S$ system. In the CIECAM02 system, the transition to $L M S$ values is given by the color values in CIE $X Y Z$ :

$$
\left[\begin{array}{c}
L \\
M \\
S
\end{array}\right]=\overrightarrow{\mathrm{M}}_{C A T 02}\left[\begin{array}{c}
X \\
Y \\
Z
\end{array}\right], \quad \overrightarrow{\mathrm{M}}_{C A T 02}=\left[\begin{array}{rrr}
0.7328 & 0.4296 & -0.1624 \\
-0.7036 & 1.6975 & 0.0061 \\
0.0030 & 0.0136 & 0.9834
\end{array}\right],
$$

where the transition to $X Y Z$ was previously made in the image using the MATLAB rgb2xyz function, and similarly for the white point. Here and further, the column vector will be indicated by an arrow " $\rightarrow$ ", the rows by a reverse arrow " $\longleftarrow$ ", and the matrices by a double arrow " $\leftrightarrow$ " above the symbol.

Then, following the von Kries hypothesis, the adapted three-stimulus $L_{c} M_{c} S_{c}$ responses representing consistent color stimuli in the reference viewing conditions (equal-energy illuminator) are determined by the expressions:

$$
L_{c}=\left(\frac{D}{L_{W}}+(1-D)\right) L, M_{c}=\left(\frac{D}{M_{W}}+(1-D)\right) M, S_{c}=\left(\frac{D}{S_{W}}+(1-D)\right) S,
$$

where the coefficient of the adaptation level $D$ is calculated according to the equations:

$$
D=F\left(1-0.28 \mathrm{e}^{-\left(L_{A}+42\right) / 92}\right),
$$

$F=0.9$ as the dim surround for computer display.

After adaptation, by going to CIE $X Y Z$ and back, the cone responses are converted to the HuntPointer-Estévez space that more closely represent cone responsivities:

$$
\left[\begin{array}{c}
L^{\prime} \\
M^{\prime} \\
S^{\prime}
\end{array}\right]=\overrightarrow{\mathrm{M}}_{H}\left[\begin{array}{c}
X_{c} \\
Y_{c} \\
Z_{c}
\end{array}\right]=\overrightarrow{\mathrm{M}}_{H} \overrightarrow{\mathrm{M}}_{C A T 02}^{-1}\left[\begin{array}{c}
L_{c} \\
M_{c} \\
S_{c}
\end{array}\right], \overrightarrow{\mathrm{M}}_{H}=\left[\begin{array}{rrr}
0.38971 & 0.68898 & -0.07868 \\
-0.22981 & 1.18340 & 0.04641 \\
0.00000 & 0.00000 & 1.00000
\end{array}\right] \text {. }
$$

The post-adaptation cone responses (for both the sample and the white) are then calculated using equations:

$$
L_{a}^{\prime}=\frac{4\left(F_{L} L^{\prime}\right)^{0.42}}{27.13+\left(F_{L} L^{\prime}\right)^{0.42}}+0.001, M_{a}^{\prime}=\frac{4\left(F_{L} L^{\prime}\right)^{0.42}}{27.13+\left(F_{L} L^{\prime}\right)^{0.42}}+0.001, S_{a}^{\prime}=\frac{4\left(F_{L} L^{\prime}\right)^{0.42}}{27.13+\left(F_{L} L^{\prime}\right)^{0.42}}+0.001,
$$

where $F_{L}=k^{4} L_{A}+0.1\left(1-k^{4}\right)^{2}\left(5 L_{A}\right)^{1 / 3}$ is the luminance-level adaptation factor, $k=\left(5 L_{A}+1\right)^{-1}$.

The obtained values uniquely determine the parameters of the color perception of $J C H$. However, from the point of view of the task set in the work, their calculation is not of interest. We simulate the light field in LI in the DIALux evo program, get a synthetic image and the maximum luminance value in the $L_{\max }$ scene. Let us take the average luminance of the image for the luminance of the adaptation $L_{A}$. Then using (1)-(5), we can get the visual perception of the image. Nevertheless, as mentioned earlier, we will display the image on the computer display, the average luminance of $L_{A D}$, and the white point is $X_{W D}, Y_{W D}, Z_{W D}$. Using the same ratios (1)-(5), but in the reverse sequence, we can recalculate the pixel colors of the image $R_{a}, G_{a}, B_{a}$, which preserved the parameters of the visual perception of the lighting system $\mathrm{JCH}$ and adapted for output to the computer display. 


\section{3D model of theater stage lighting}

The initial data for the task is the layout of the premises of the Vladimir Vysotsky Theater. The DIALux evo software was chosen to implement this model, which includes sufficient functionality for calculating the necessary parameters, visualization, and a large set of lighting devices. Figure 1 shows a visualization of the three-dimensional view of the resulting model. The room's dimensions were calculated based on available information about the number of seats in the hall: eight rows of seats on the first floor of the room and three rows of seats on the balcony.

For illuminating the foreground of the stage and the actors, lighting devices were built on the hall's front part - the balcony. Artemide PICTO LED HIGH FLUX 4000K SPOT ND/AD NERO lighting devices were chosen as spotlights. Lighting fixtures were added to the stage side for the side lighting of the actors and the scenery. The searchlights of the Siemens STAGE PROJECTOR WITH ARM company were chosen as lighting devices. Lighting fixtures were added to the upper part of the stage to illuminate the background. The searchlights of the Siemens MEGAFOCUS company were chosen as lighting devices. All the floodlights added to the model have lamps with a lighting temperature of $3000-4000 \mathrm{~K}$. This choice is because the sunset atmosphere assumes low illumination in warm shades.

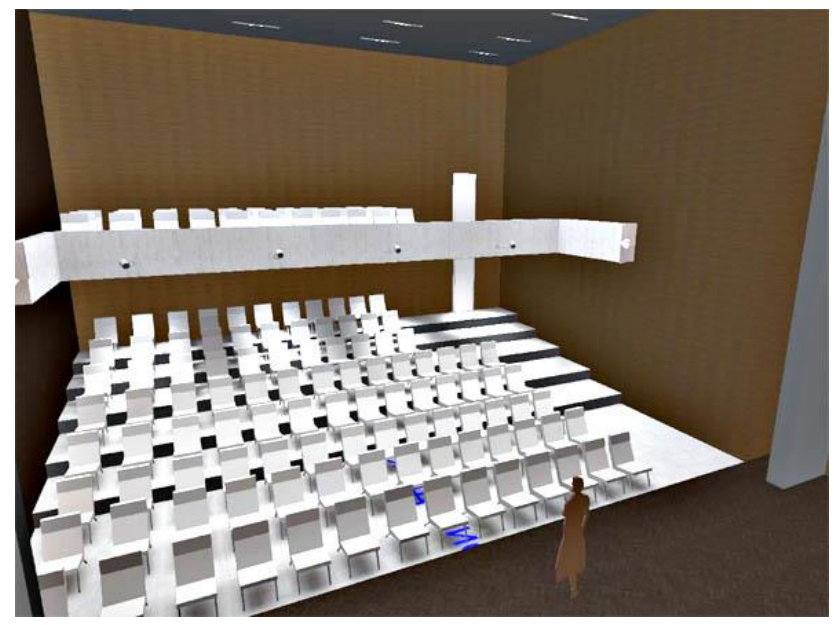

Figure 1: 3D model of Vladimir Vysotsky theater.

The sunset atmosphere was created using references taken from the Internet for the request sunset, two of which are shown in Figure 2. Sunset is not a one-time phenomenon. It takes a considerable period, changing from a bright orange color through red to blue and purple. At sunset, the leading color solutions are the predominance of red, red shades if we are talking about early sunset when the sun has not yet set. If we are talking about a later sunset, when the sun has almost set, then the color solutions are replaced by pink shades from the sun side and the predominance of purple and blue shades on the other sunset side.

In this work, several variants of atmospheric lighting were created at different times of sunset. To creating the illusion of the sun shining through the window and adding a textured shadow on the wall, the lighting included a side spotlight placed on the side of the stage. The color filter of this spotlight had a red hue of different saturation.

For illuminating the actor on the stage, front lighting was used with a searchlight placed on the balcony side as the artificial sun (in this work, on the left). For such a searchlight, color filters with the same red shades were used as for side lighting.

The background lighting was created by overhead lighting placed on the top of the stage. In this case, the color filters were of different shades depending on the location of the sun - the farther away from the left part of the scene, the bluer shades prevailed, the closer to the left part, the more yellow and red shades prevailed. 

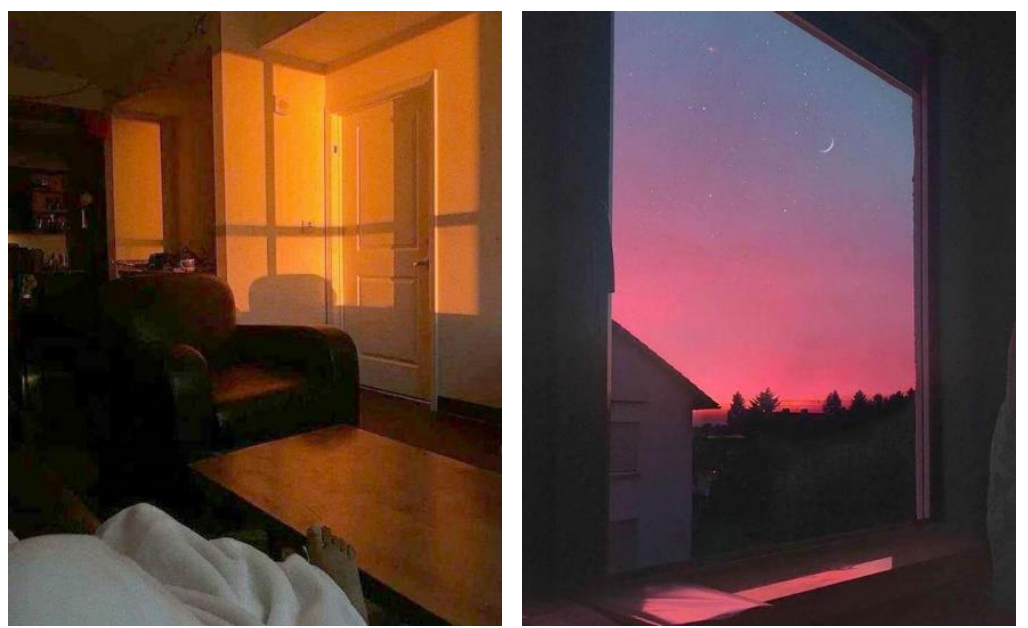

Figure 2: Sunset Reference Examples.

Figure 3 shows the final images of the theatrical stage lighting options model with a sunset atmosphere from early No. 1 to late No. 7.

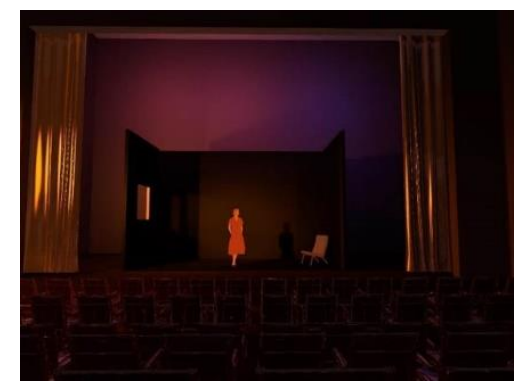

Variant 1

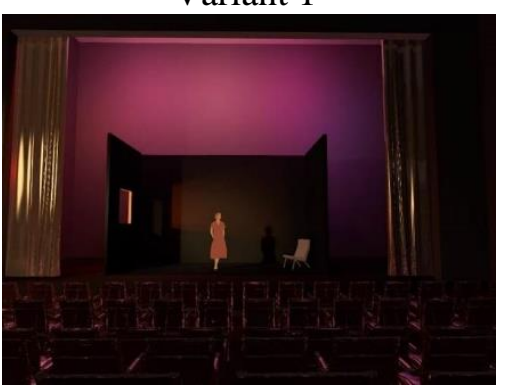

Variant 4

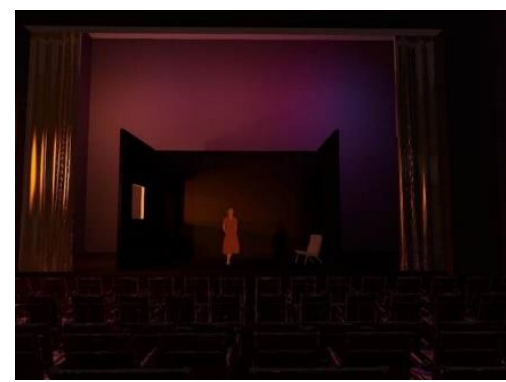

Variant 2

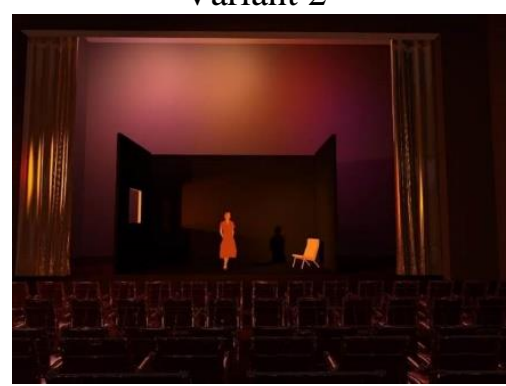

Variant 5

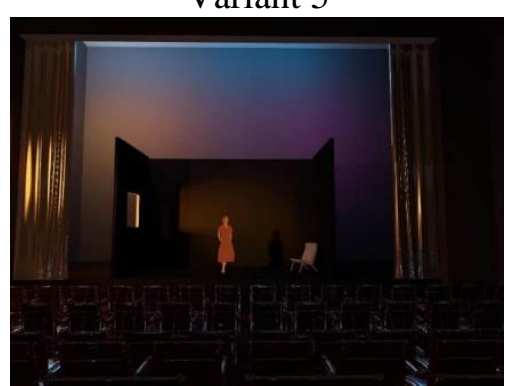

Variant 7

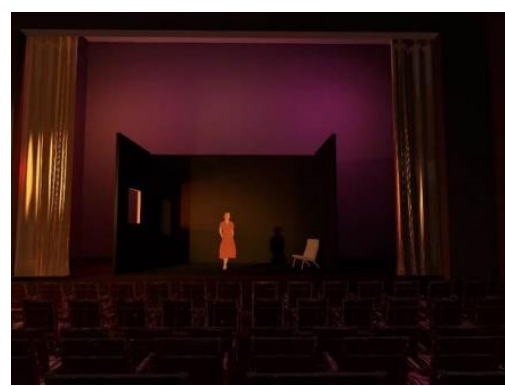

Variant 3

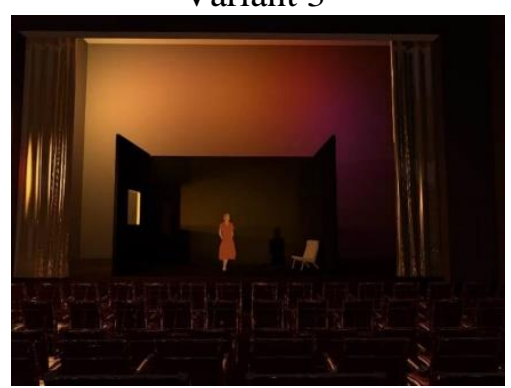

Variant 6

Figure 3: Visualization of the lighting of the scene with the sunset atmosphere.

To create a realistic image, a program was used that implements the CIECAM02 model algorithm described above. The maximum luminance in the lighting scene according to the calculation in the DIALux evo software is $L_{A}=10 \mathrm{~cd} / \mathrm{m}^{2}$. Further, a reverse recalculation was performed according to the ICC profile of the computer display. The results of the images to adapt to the luminance of the display are shown in Figure 4. 
The obtained images were evaluated for similarity in the feeling of sunset by the method of experimental estimates. For conducting surveys, the following sites were used: https://forms.gle/3KhCJ6mwFZobbuJZ7 and https://forms.gle/oB5z5szssRDAydQ18. To solve this problem, a scale was introduced by which images were evaluated. The image with the highest number of positive results is considered the best alternative. In the survey, images were presented in an order unknown to experts before and after processing. The scale for evaluation includes four options [13]:

1. Nothing like (the farthest result from lighting with a sunset atmosphere).

2. Not very similar (there are differences from the sunset atmosphere).

3. A little similar (there are shortcomings, but they are not critical).

4. Remarkably similar (the atmosphere is most suitable for sunset).

Experts are invited to choose one of the most suitable options for each of the proposed images.

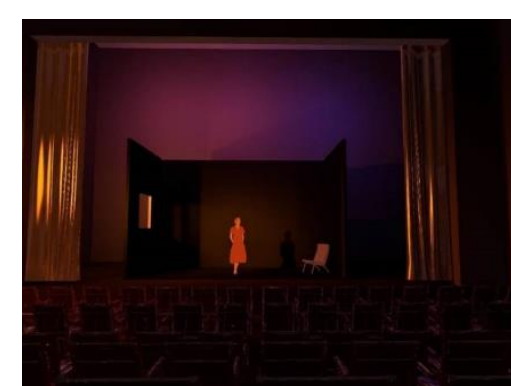

Variant 1

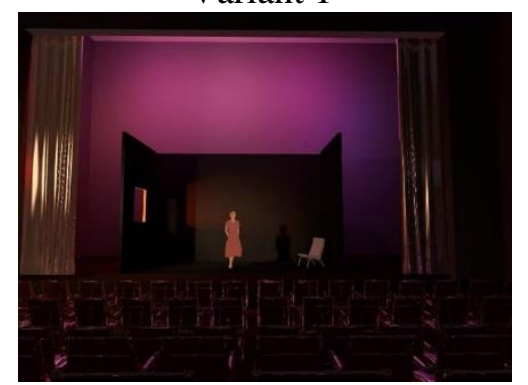

Variant 4

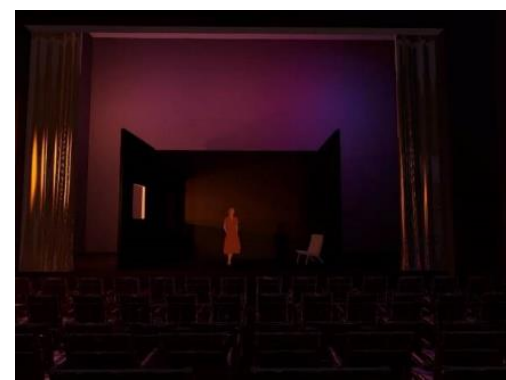

Variant 2

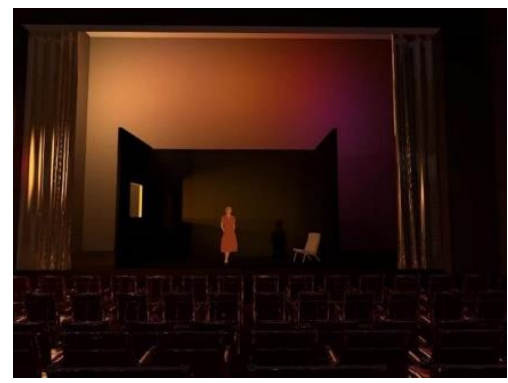

Variant 5

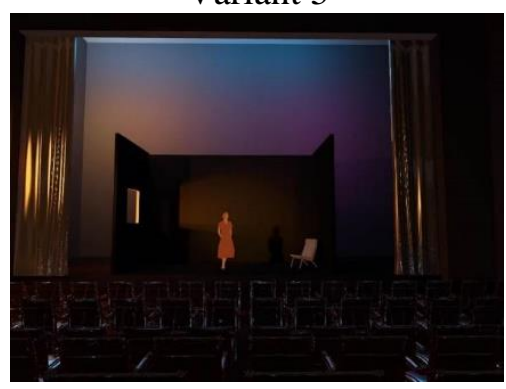

Variant 7

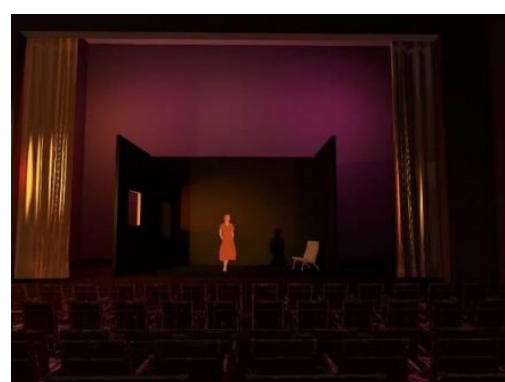

Variant 3

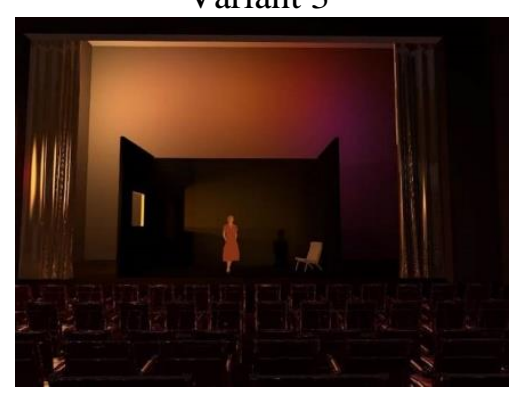

Variant 6

Figure 4: Images when observed at adapting to the display luminance.

Figure 5 shows the response diagrams for each of the image variants before and after transformations.

After summing up the survey results, the best images before the conversion turned out to numbers $1,5,6$, and after the conversion, the image became the best option number 6 . As can be seen from the graph, the total number of responses "Remarkably similar" decreased, which indicates that after processing, the image becomes less suitable for lighting a scene with a sunset atmosphere. In general, the number of answers "Not very similar "and "A little similar" prevails, so we can conclude that the images obtained are not quite suitable for this stage lighting. Therefore, it was decided to conduct a second experiment in which new modified lighting options will be created based on expert comments.

After the first survey, the most similar images for the sunset from the proposed options were identified. An additional survey was created to determine the main points (the selection of color, brightness, and saturation of lighting) that affect experts' decisions. The survey included the three best 
images. The questions included a scale from 1 to 8 , where 1 is not similar, 8 is very similar. The experts were asked to evaluate the selection of the lighting color scheme, brightness, and color saturation on a scale. Also, in addition to each image, it was suggested to add the general opinion of each expert about what is missing for the "perfect sunset" in the image. Let us look at the survey results for the best one. Variant number 6 of the first experiment was selected (Figure 6).
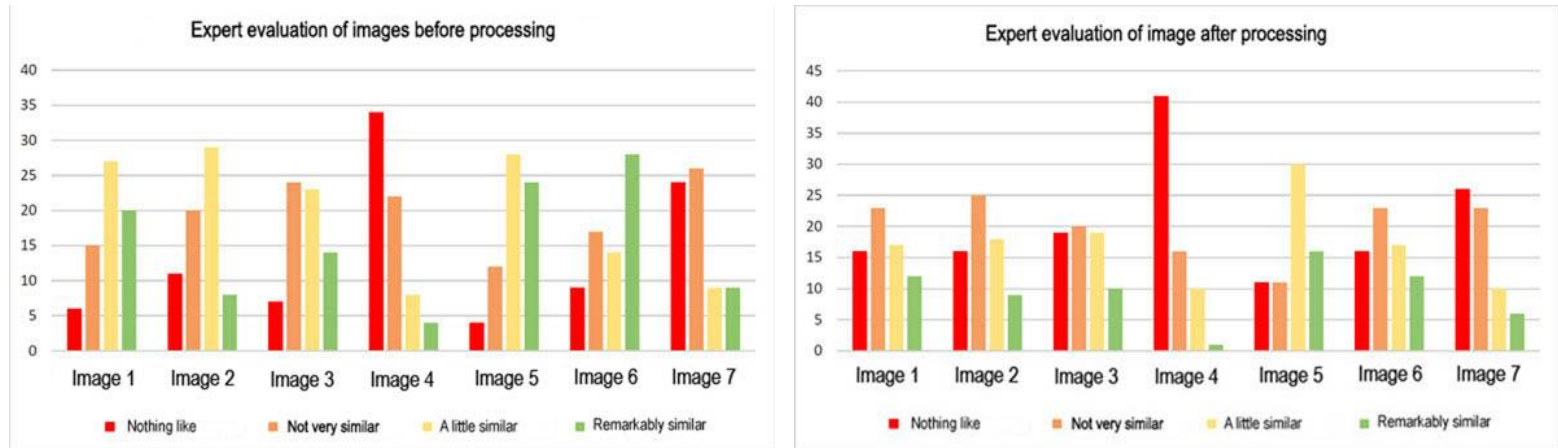

Figure 5: Diagram of the distribution of expert assessment responses.

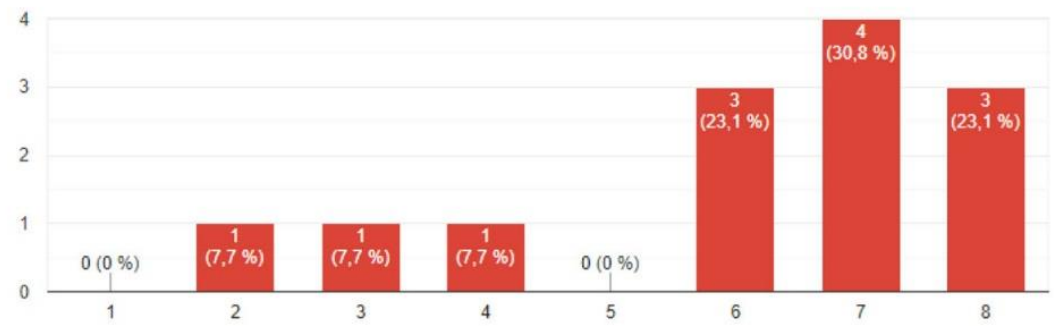

Chromacity

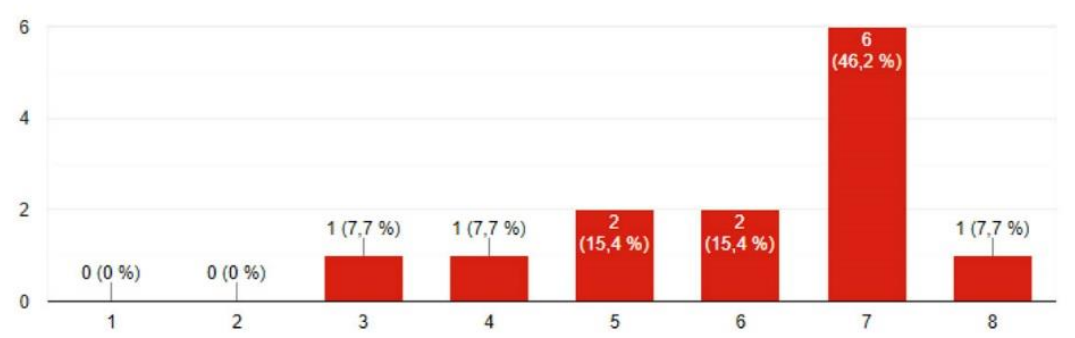

Lightness

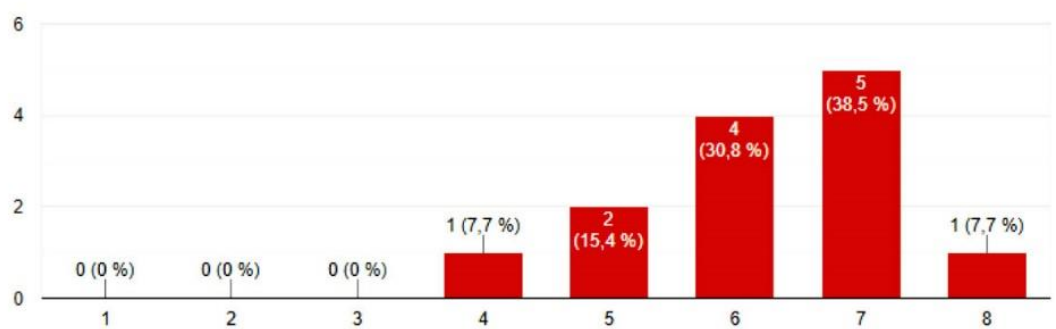

Saturation

Figure 6: Diagrams of the similarity of the best variant No. 6 with the sunset.

Expert answers on the graphs have a normal distribution. Based on the results, we can conclude that the brightness and saturation almost meet experts' expectations. According to the color solution in the last question, experts believed there are not enough purple shades in the sky. 
After processing the survey results, three versions of the images were finalized based on experts' opinions. Figure 7 shows the changes to variant number 6 . Purple shades were added in the upper right corner. The color saturation was slightly increased. In the center, Figure 7 shows the changes to variant number 5 - uniformity in shades was added. The primary color of the sunset is pink. After surveys, it was found out that red colors in the lighting positively affect the perception of the sunset atmosphere. Therefore, it was decided to make a third variant with the main color in red shades. For the contrast between the actor and the scenery, the luminance of the front lighting was added. The visualization of the new stage lighting is shown in Figure 7 on the right.

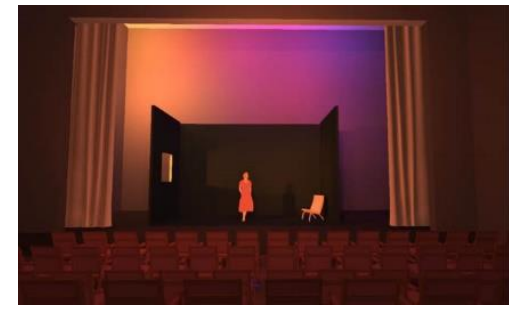

Variant 6

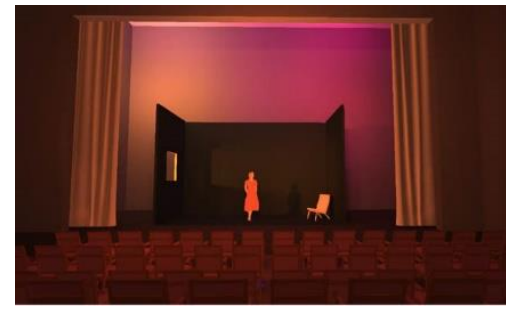

Variant 5

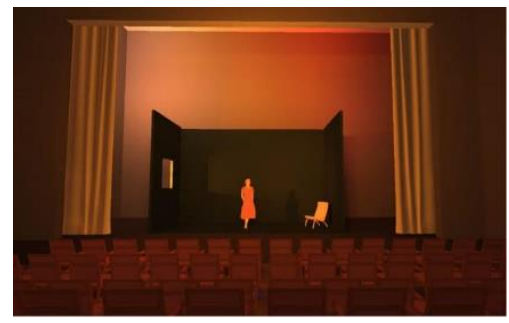

New variant

Figure 7: Variants of improved stage lighting.

On all models, the luminance index of lighting devices was made at $100 \%$ since it was previously found out that after converting images, they become dimmer. To achieve the desired result, it is necessary to make slightly illuminated images in the software.

After receiving the results of the models, the final realistic images of the stage lighting were obtained. These images are shown in Figure 8.

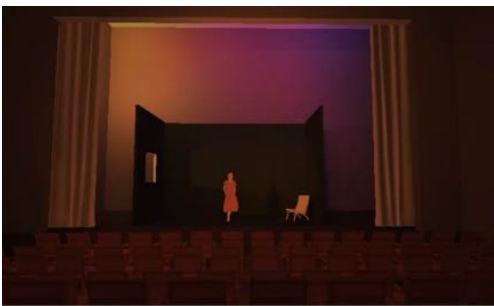

Variant 6

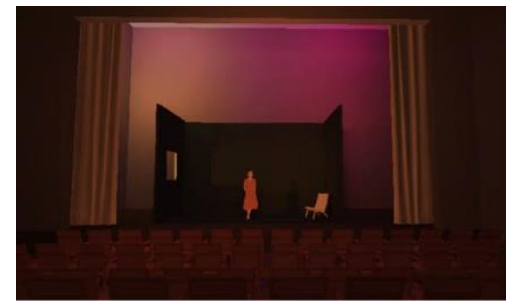

Variant 5

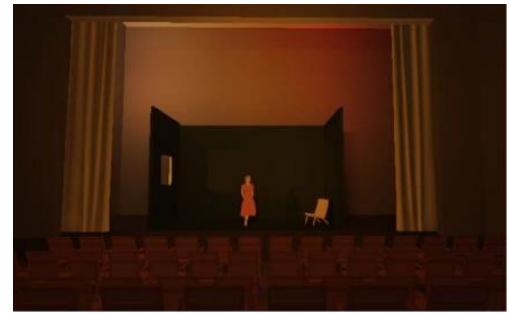

New variant

Figure 8: Variants of improved stage lighting are adapted to the observation conditions on display.

\section{Conclusions}

Based on the results obtained and the survey conducted, the following recommendations were formed for the design of stage lighting in the DIALux evo software:

1. When designing stage lighting of the sunset atmosphere, it is recommended to use mainly red and pink shades of color filters on installed spotlights.

2. It is recommended not to use blue and purple shades in large quantities, as they are associated with night lighting.

3. To get the desired result in real life, it is recommended to design the lighting in the software brighter to get the desired result.

4. For the realistic image synthesis when using CIECAM02, it is necessary to use information about the luminance over the lighting scene in absolute units from the LI simulation program and the display ICC profile.

It seems that the analysis of the realistic modeling of the lighting of the scene with the feeling of sunset allows us to assert that computer graphics programs need to be supplemented with the CIECAM02 color reproduction model before displaying a synthetic image on the display screen. This 
will allow you to realistically evaluate the light and color design of the projected lighting system based on its image.

\section{Acknowledgements}

We want to thank all graduate students and students of the Department of Computer-aided Design and Design at the "National University of Science and Technology "MISiS" and the Department of Light Engineering at the "National Research University "MPEI" for participating in the expert assessment surveys of images based on their similarity to the feeling of sunset.

\section{References}

[1] A Colour Appearance Model for Colour Management Systems: CIECAM02, CIE Publication 159, CIE Central Bureau, Vienna, Austria, 2004.

[2] M.D. Fairchild, Color Appearance Models, 2nd. ed., Wiley, Chichester, 2005.

[3] C. Ma, L. Ou, An initial study of colour appearance in virtual reality, in: Proceedings of 29th CIE Session, Washington D.C., USA, 2019, pp.32-35. doi: 10.25039/x46.2019.OP05.

[4] T.H. Phung, et al. Applying an image colour appearance model for simple self-luminous scenes, Proceedings of 29th CIE Session, Washington D.C., USA, 2019, pp. 49-54. doi:10.25039/x46.2019.0P08.

[5] C. Li, Z. Li, Z. Wang, Y. Xu, M.R. Luo, G. Cui, M. Melgosa, M.H. Brill, M. Pointer, Comprehensive color solutions: CAM16, CAT16, and CAM16-UCS, Color Res. Appl., 42 (2017) 703-718.

[6] I. Newton, Optics, or a Treatise of the Reflections, Refractions, Inflections and Colors of Light, Wm. Innys, London, 1730.

[7] T. Young, On the Theory of Light and Colours, the Bakerian Lecture, Phil. Trans. 92 (1802) 12 48.

[8] H. Helmholtz, On the Theory of Compound Colours, Phil. mag. 4 (1852) 519-534.

[9] H.G. Grassmann, On the Theory of Compound Colours, Philosophical Magazine 7 (1854), 254264.

[10] J.C. Maxwell, Experiments on Colour, as perceived by the Eye, with remarks on Colour-Blindness, in: The Scientife Papers of James Clerk Maxwell, Vol. 1, Cambridge Univ. Press, Cambridge, 1890.

[11] CIE 1931: Colorimétrie, Resolutions 1-4, in: Recueil des travaux et compte rendu des séances, Huitiéme Session Cambridge, The National Physical Laboratory Teddington Herausgeber, 1931, pp. 19-29.

[12] J. von Kries, Chromatic adaptation, Festschrift der Albrecht-Ludwig-Universitat, Freiburg, 1902.

[13] V.P. Budak, E.I. Ilyina, Construction of a psychophysical scale of visual comfort of lighting based on a neural network: preparation of the experiment, Light \& Engineering 29 (2021) 114-122. doi:10.33383/2020-067. 